\title{
Parametric Analysis of Heat Transfer in Gypsum Wallboard Partitions
}

\author{
MORGAN BRUNS and KULDEEP PRASAD \\ Fire Research Division \\ National Institute of Standards and Technology (NIST) \\ Gaithersburg, Maryland, USA
}

\begin{abstract}
One-dimensional heat transfer in gypsum wallboard partitions is studied using sensitivity analysis and calibration by error minimization. Analysis of an existing heat and mass transfer model indicates that mass transfer and condensation of water vapor are not of primary importance in predicting board temperatures. A computationally efficient and robust heat transfer model is developed for predicting temperatures in gypsum wallboard partitions. Kinetic parameters are calibrated by error minimization with respect to literature thermogravimetric analysis (TGA) data. Additional thermophysical parameters are calibrated by error minimization with respect to literature ASTM E119 furnace test data. It is found that the calibrated heat transfer model is capable of predicting board temperatures given large thermal conductivities and specific heats at high temperatures. It is hypothesized that these observations imply the need to account for porous media radiation, temperature varying specific heats, and calcium carbonate decomposition in future models. Local sensitivity analysis reveals that board temperatures are most sensitive to initial density, thermal conductivity at moderately high temperatures $\left(\sim 800{ }^{\circ} \mathrm{C}\right)$, and the activation energy of the dehydration reactions. Conversely, model predictions are relatively insensitive to dehydration reaction pre-exponentials and low-temperature heat capacities.
\end{abstract}

KEYWORDS: modeling, fire resistance, heat transfer, gypsum, sensitivity analysis, optimization

\section{NOMENCLATURE}

$A_{j} \quad$ pre-exponential of reaction $j(1 / \mathrm{s})$

$c_{p}$ heat capacity $(\mathrm{J} / \mathrm{kg} / \mathrm{K})$

$e_{2} \quad$ sum of squared relative errors

$D$ mass diffusivity $\left(\mathrm{m}^{2} / \mathrm{s}\right)$

$E_{j} \quad$ activation energy of reaction $j(\mathrm{~kJ} / \mathrm{mol})$

$h$ heat transfer coefficient $\left(\mathrm{W} / \mathrm{m}^{2} / \mathrm{K}\right)$

$k$ thermal conductivity $(\mathrm{W} / \mathrm{m} / \mathrm{K})$

$L_{b} \quad$ gypsum board thickness (m)

$L_{g} \quad$ air gap thickness (m)

$M_{i} \quad$ molecular weight of species $i(\mathrm{~g} / \mathrm{mol})$

$q^{\prime \prime} \quad$ heat flux $\left(\mathrm{W} / \mathrm{m}^{2}\right)$

$\dot{q}^{\prime \prime \prime} \quad$ volumetric heating rate $\left(\mathrm{W} / \mathrm{m}^{3}\right)$

$r_{j} \quad$ rate of reaction $j\left(\mathrm{~kg} / \mathrm{m}^{3} / \mathrm{s}\right)$

$R \quad$ gas constant $(\mathrm{J} / \mathrm{mol} / \mathrm{K})$

$S \quad$ sensitivity

$t$ time (s)

$T$ temperature $\left({ }^{\circ} \mathrm{C}\right)$

$w \quad$ normalized mass

$x \quad$ distance from exposed surface (m)

$y \quad$ free model parameter

$Y_{1,0} \quad$ initial mass fraction of dihydrate

$\begin{array}{ll}\text { Greek } \\ \Delta h_{j} \quad \text { heat of reaction } j(\mathrm{~kJ} / \mathrm{kg}) \\ \varepsilon & \text { emissivity } \\ \kappa & \text { permeability }\left(\mathrm{m}^{2}\right) \\ \lambda_{j} & \text { rate constant of reaction } j(1 / \mathrm{s}) \\ \rho & \text { composite density }\left(\mathrm{kg} / \mathrm{m}^{3}\right) \\ \rho_{i} & \text { component } i \text { bulk density }\left(\mathrm{kg} / \mathrm{m}^{3}\right) \\ \sigma & \text { Stefan-Boltmann constant }\left(\mathrm{W} / \mathrm{m}^{2} / \mathrm{K}^{4}\right) \\ \phi & \text { porosity } \\ \Omega & \text { feasible parameter space } \\ \text { Subscripts } \\ 0 & \text { initial state } \\ 1 b & \text { back of first board } \\ 1 f & \text { front of first board } \\ 2 b & \text { back of second board } \\ 2 f & \text { front of second board } \\ c & \text { convective heat flux } \\ r & \text { radiative heat flux } \\ \infty & \text { ambient condition }\end{array}$

INTRODUCTION

Fire spread in buildings may be impeded by fire-rated partitions. The fire resistance of a partition is typically quantified by the time taken for the back of the partition to reach a certain temperature when the front of 
the partition is exposed to a standard time-temperature curve. Performance is typically rated in furnace tests using the standard ASTM E119 exposure [1]. Unfortunately, these ratings do not correlate well with actual performance in building fires. In particular, the ASTM E119 temperature curve was designed to represent cellulosic fuels that are not representative of modern building fire fuel sources [2]. A possible solution to this problem is to transition to a performance-based design paradigm. Such a shift requires the development of accurate models for heat transfer across building partitions. In this paper, a one-dimensonal heat transfer model is evaluated for its effectiveness in predicting temperatures across an unloaded gypsum wallboard partition.

Several models of heat transfer in gypsum wallboard partitions have been proposed in the literature. Mehaffey et al. [3] proposed a two-dimensional heat transfer model formulated in terms of enthalpy. Sultan [4] developed a one-dimensional model for steel-stud partitions. This model was essentially of the same form as that of Mehaffey et al. with the exception of the use of temperature dependent convection coefficients. In an effort to improve model accuracy and reliability, Kukuck et al. [5], [6] developed a model including mass transfer and condensation of water vapor. Water vapor velocities were determined from Darcy's law. The model was found to predict furnace data fairly well, but it was not clearly demonstrated that the inclusion of mass transfer and condensation resulted in a significant improvement over pure heat transfer models. In an attempt to systematically analyze the importance of mass transfer and condensation, Weber [7] compared three models of transport in a single gypsum board: (1) pure heat conduction, (2) heat/mass transfer, and (3) heat/mass transfer plus condensation. The pure heat transfer model predicted time-temperature curves fairly well. Addition of a mass transfer model led to a marginal improvement in predicting certain portions of the time-temperature curve. The third model included condensation of water vapor, and seemed to provide little improvement over predictions from the heat/mass transfer model.

Much of the error in the preceding models is likely attributable to model parameter uncertainty. The approach of this paper is to explore the sensitivity of model results to material properties. Such an analysis is valuable for identifying which properties need to be measured more precisely and whether or not heat transfer alone is sufficient for modeling the fire resistance of gypsum wallboard partitions. To this end, three issues are addressed. First, the importance of including mass transfer and water vapor condensation in gypsum wallboard partitions is investigated by model prediction comparisons and local sensitivity analysis. Second, the feasibility of a pure heat conduction model is tested by calibrating such a model to experimental furnace test data. Third, the relative importance of model parameters are ranked based on a sensitivity analysis of the calibrated model.

\section{IMPORTANCE OF MASS TRANSFER AND CONDENSATION OF WATER VAPOR}

Commercial gypsum wallboards are predominantly composed of calcium sulfate dihydrate. As the material is heated, the chemically bound water is released endothermically as a result of two dehydration reactions

$$
\begin{aligned}
& \mathrm{CaSO}_{4} \cdot 2 \mathrm{H}_{2} \mathrm{O} \stackrel{\lambda_{1}}{\longrightarrow} \mathrm{CaSO}_{4} \cdot \frac{1}{2} \mathrm{H}_{2} \mathrm{O}+\frac{3}{2} \mathrm{H}_{2} \mathrm{O}(\mathrm{g}) \\
& \mathrm{CaSO}_{4} \cdot \frac{1}{2} \mathrm{H}_{2} \mathrm{O} \stackrel{\lambda_{2}}{\longrightarrow} \mathrm{CaSO}_{4}+\frac{1}{2} \mathrm{H}_{2} \mathrm{O}(\mathrm{g})
\end{aligned}
$$

where $\lambda_{1}$ and $\lambda_{2}$ are rate constants. The associated dehydration reactions absorb a significant amount of energy thereby reducing the heat transfer rate through the board. Water vapor is free to flow through the porous gypsum and condense in colder regions of the board. The condensed water can flow due to capillary pressure. Eventually temperatures will become sufficiently high such that all of the moisture is released from the board.

As was discussed in the previous section, it is unclear whether water vapor transport and condensation significantly influence the temperature distribution in the partition. To investigate this question, the model of Kukuck [5] was used to simulate temperatures in a gypsum wallboard partition with and without mass transfer and condensation in an ASTM E119 furnace test. This model was chosen because it includes mass transfer and condensation of water vapor. Material properties were taken from [5]. Plots of the temperatures at the backs of the exposed, $T_{1 b}$, and unexposed boards, $T_{2 b}$, are given in Fig. 1 for the three models along with experimental data from Takeda and Mehaffey [8]. It is seen that there is very little noticeable difference in FIRE SAFETY SCIENCE-PROCEEDINGS OF THE ELEVENTH INTERNATIONAL SYMPOSIUM pp. 598-611 


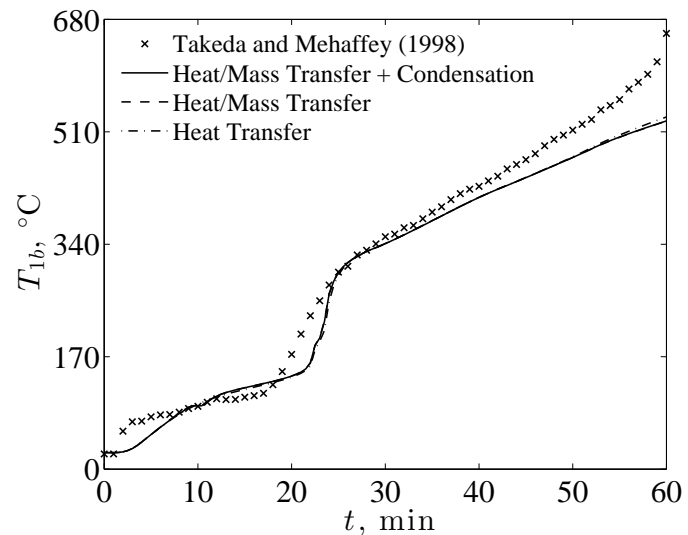

(a)

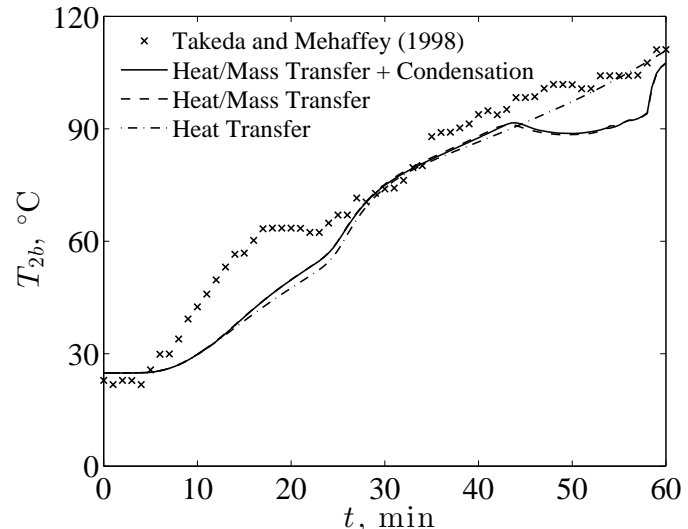

(b)

Fig. 1. Temperatures at the back of the exposed (a) and unexposed (b) gypsum boards using three physical models of an ASTM E119 furnace test.

the model predictions at the back of the exposed board. At the back of the unexposed board, both models with mass transfer predict a significant dip in $T_{2 b}$ beginning at around $42 \mathrm{~min}$. This dip is is not observed in literature furnace test data (e.g., [3], [4], or [8]). Aside from this temporary drop in temperature, there is little difference between the predictions of the heat transfer model and the models including mass transfer. Also, in agreement with the results of Weber [7], there is no significant difference resulting from the addition of condensation to the heat/mass transfer model.

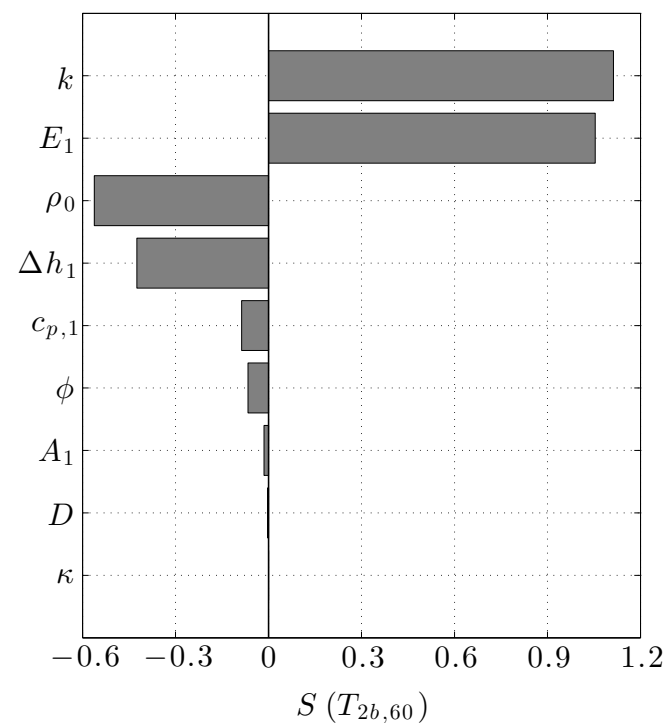

Fig. 2. Sensitivities of temperature at the back of the unexposed board at $60 \mathrm{~min}$ with respect to several parameters in the heat/mass transfer model.

To further investigate the importance of mass transfer, a local sensitivity analysis of the model of Kukuck [5] was performed. Computation of sensitivities requires an appropriate quantity of interest. For the case of wallboard partition furnace tests, fire resistance ratings typically specify maximum back wall temperatures at one hour [1]. The sensitivity of the predicted value of $T_{2 b}$ at 60 min with respect to model parameter $y$ is 
defined as

$S\left(T_{2 b, 60}\right)=\frac{\tilde{y}}{\tilde{T}_{2 b, 60}} \frac{\partial T_{2 b, 60}}{\partial y}$

where $\tilde{y}$ is the nominal value of parameter $y$ and $\tilde{T}_{2 b, 60}$ is the temperature predicted with nominal material properties. All material properties except $y$ are held constant at their nominal values in computing $S\left(T_{2 b, 60}\right)$. Dimensionless sensitivities were chosen in order to better compare the relative influence of the various model parameters. The sensitivities were approximated by converged finite differences and are plotted in Fig. 2 for nine parameters. The properties considered were thermal conductivity $(k)$, activation energy of the first dehydration reaction $\left(E_{1}\right)$, initial density $\left(\rho_{0}\right)$, heat of the first dehydration reaction $\left(\Delta h_{1}\right)$, specific heat capacity of the calcium sulfate dihydrate $\left(c_{p, 1}\right)$, porosity $(\phi)$, pre-exponential of the first dehydration reaction $\left(A_{1}\right)$, mass diffusivity $(D)$, and permeability $(\kappa)$. It is seen that the back wall temperature is most sensitive to the thermal conductivity and the activation energy of the first dehydration reaction. The model prediction shows almost zero sensitivity to the mass transfer properties (i.e., the mass diffusivity and permeability).

The results of this section indicate that mass transfer and condensation are not of primary importance in predicting temperatures in gypsum wallboard partitions. However, the evidence is not conclusive because of model parameter uncertainty. In particular, the computed sensitivities are dependent on the assumed nominal material. The assumed nominal properties are uncertain, and it is possible that another choice of nominal values would result in the model output showing significant sensitivity to the mass transfer and condensation properties. In the remainder of this paper, the question of whether a pure heat conduction model is sufficient for predicting wallboard partition temperatures is investigated. To this end, a computationally efficient heat transfer model is developed in the next section.

\section{HEAT TRANSFER MODEL}

The model developed in this section is similar to that of Sultan [4]. Instead of an enthalpy formulation, conservation of energy is expressed in terms of temperature. Such a formulation is more appropriate since an explicit kinetic model for the dehydration reactions is included. Additionally, more sophisticated heat transfer coefficient correlations are used, and the exposed surface boundary condition is treated as an imposed temperature. The assumption of an exposed surface temperature boundary condition was driven by uncertainty about the thermal conditions in the furnace. Sultan suggested that a forced convection coefficient was more appropriate than the natural convection coefficient used in his paper.

In comparison with the model of Kukuck [5], the following model does not account for mass transfer and condensation. In terms of computational performance, the code was designed to be less general but more efficient than Kukuck's code.

\section{Solid Phase Governing Equations}

The composition of the gypsum is assumed to change according to the two reactions Eqs. 1 and 2. Note that the dehydrated water is assumed to go to the gaseous state so that the heats of reaction will include contributions from vaporization. Other reactions considered in the literature have not been included here. The reaction model of Kukuck [5] includes a phase change reaction from soluble to insoluble anhydrous calcium sulfate at around $420{ }^{\circ} \mathrm{C}$. Since this reaction does not change the mass and it is accompanied by a relatively small heat of reaction, it will not be included in the model. Another possible reaction is the decomposition of calcium carbonate ( $\sim 10 \%$ by mass of the gypsum) at around $800^{\circ} \mathrm{C}$ [10]. Calcium carbonate decomposition was not included because most literature models do not include this reaction.

The material will be modeled as consisting of three components representing dihydrate, hemihydrate, and anhydrous calcium sulfate. The composition of the composite is characterized by three bulk densities: $\rho_{1}$ for the dihydrate, $\rho_{2}$ for the hemihydrate, and $\rho_{3}$ for the anhydrous calcium sulfate. The composite density is the sum of the bulk densities, or $\rho=\rho_{1}+\rho_{2}+\rho_{3}$. 
The time rate of change of component densities are

$\frac{\partial \rho_{1}}{\partial t}=-\lambda_{1} \rho_{1}$

$\frac{\partial \rho_{2}}{\partial t}=\left(M_{2} / M_{1}\right) \lambda_{1} \rho_{1}-\lambda_{2} \rho_{2}$

$\frac{\partial \rho_{3}}{\partial t}=\left(M_{3} / M_{2}\right) \lambda_{2} \rho_{2}$

where $M_{1}=172 \mathrm{~g} / \mathrm{mol}, M_{2}=145 \mathrm{~g} / \mathrm{mol}$, and $M_{3}=136 \mathrm{~g} / \mathrm{mol}$ are the molecular weights of the components. It is assumed that the reaction rates have an Arrhenius temperature dependence

$\lambda_{j}=A_{j} \exp \left(-E_{j} / R T\right), \quad j=1,2$

where $A_{j}$ and $E_{j}$ are the pre-exponential and activation energies for reactions 1 and 2.

The one-dimensional energy equation for a reacting solid is

$\rho c_{p} \frac{\partial T}{\partial t}=\frac{\partial}{\partial x}\left(k \frac{\partial T}{\partial x}\right)+\dot{q}^{\prime \prime \prime}$

where $\dot{q}^{\prime \prime \prime}$ is the net volumetric energy production due to chemical reactions. Thermal conductivity, $k$, will be modeled as a piece-wise function of temperature only, but the volumetric heat capacity, $\rho c_{p}$, is composition dependent. Heat capacity in composite materials is typically modeled as additive on a mass basis such that

$\rho c_{p}=\rho_{1} c_{p, 1}+\rho_{2} c_{p, 2}+\rho_{3} c_{p, 3}$

where $c_{p, i}$ denotes the mass specific heat capacity of pure species $i$.

The volumetric energy source term depends on the reaction rates and heats of reaction. For the reaction model considered in this paper, the source term is computed as

$\dot{q}^{\prime \prime \prime}=-\lambda_{1} \rho_{1} \Delta h_{1}-\lambda_{2} \rho_{2} \Delta h_{2}$

where $\Delta h_{1}$ and $\Delta h_{2}$ are the heats of reactions 1 and 2, respectively. For both reactions, $\Delta h_{j}>0$ since the dehydration reactions are endothermic.

\section{Geometry and Boundary Conditions}

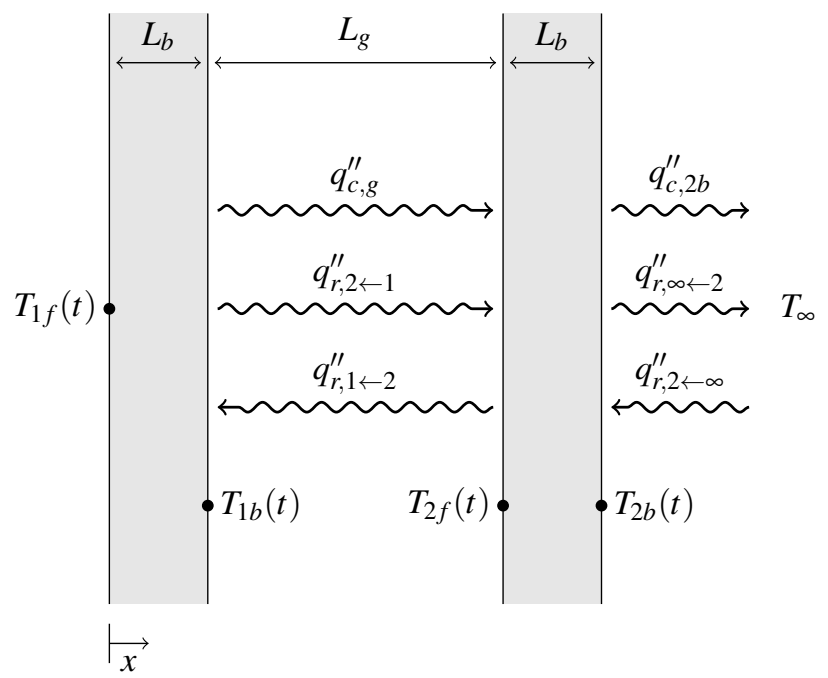

Fig. 3. Partition geometry and heat fluxes. 
A schematic of a typical gypsum wallboard partition is provided in Fig. 3. The partition consists of two boards of thickness $L_{b}$ separated by an air gap of thickness $L_{g}$. At the front of the partition, the surface is exposed to an ASTM E119 temperature curve, $T_{1 f}(t)$ - this choice is a significant approximation and is discussed in the next paragraph. Heat is conducted through the front board according to the heat transfer model of the previous section. At the back of the front board, heat is lost to the back board by convection, $q_{c, g}^{\prime \prime}$, and radiation, $q_{r, 2 \leftarrow 1}^{\prime \prime}$. Some heat from the back board is radiated back to the front board, $q_{r, 1 \leftarrow 2}^{\prime \prime}$. Heat is conducted through the back board and is eventually lost from the partition due to convection, $q_{c, 2 b}^{\prime \prime}$, and radiation exchange, $q_{r, \infty \leftarrow 2}^{\prime \prime}$ and $q_{r, 2 \leftarrow \infty}^{\prime \prime}$, with the surroundings at ambient temperature, $T_{\infty}$.

As mentioned above, the choice of a surface boundary condition is not straightforward. The ASTM E119 standard requires that furnace temperatures follow the prescribed time-temperature curve, but depending on how these temperatures are achieved and measured, different surface boundary conditions could be appropriate for different furnaces. Kukuck [5] assumed that the boundary condition was one of convection and radiation from surroundings at the ASTM E119 temperatures. However, in furnaces with burners, radiation could be from a flame temperature much higher than the measured furnace gas temperature. Also, convective heating will be dependent on the flow of gases within the furnace. One possibility for modeling the exposed surface boundary condition is to use measured heat fluxes. The surface heat fluxes on an ASTM E119 furnace were measured as a function of time and applied as model inputs by Sultan et al. [11], but such an approach is contrary to the ultimate goal of performance-based design since it requires scenario specific experiments. Since the appropriate front surface boundary condition is uncertain, the simplest alternative was chosen. In future work, the furnace could be modeled in detail to determine the thermal conditions at the front of gypsum wallboard partition. Additionally, fluid flow models could be implemented to determine the convective heat transfer within the air gap.

Solution of the condensed phase conservation equation requires two boundary conditions for each gypsum wallboard. Following the schematic of Fig. 3, these boundary conditions are as follows:

$$
\begin{aligned}
& T(x=0, t)=T_{1 f}(t) \\
& -\left.k \frac{\partial T}{\partial x}\right|_{x=L_{b}}=h_{g}\left(T_{1 b}-T_{2 f}\right)+\varepsilon \sigma\left(T_{1 b}^{4}-T_{2 f}^{4}\right) \\
& -\left.k \frac{\partial T}{\partial x}\right|_{x=L_{b}+L_{g}}=h_{g}\left(T_{1 b}-T_{2 f}\right)+\varepsilon \sigma\left(T_{1 b}^{4}-T_{2 f}^{4}\right) \\
& -\left.k \frac{\partial T}{\partial x}\right|_{x=2 L_{b}+L_{g}}=h_{2 b}\left(T_{2 b}-T_{\infty}\right)+\varepsilon \sigma\left(T_{2 b}^{4}-T_{\infty}^{4}\right)
\end{aligned}
$$

where the convective coefficients are temperature dependent and computed using correlations from ElSherbiny et al. [12] for $h_{g}$ and from Churchill and Chu [13] for $h_{2 b}$. Shorthand is used for the surface temperatures such that $T_{1 b} \equiv T\left(x=L_{b}\right), T_{2 f} \equiv T\left(x=L_{b}+L_{g}\right)$, and $T_{2 b} \equiv T\left(x=2 L_{b}+L_{g}\right)$. It is also assumed that the gypsum emissivity, $\varepsilon$, is constant with respect to temperature and composition.

Initially the partition is assumed to be at the ambient temperature, or $T(x, t=0)=T_{\infty}$. The composition at time $t=0$ is characterized by the initial mass fraction of dihydrate, $Y_{1,0}$, and the initial gypsum board density, $\rho_{0}$. It is assumed that the remainder of the material is anhydrous calcium sulfate. Thus, the initial bulk densities are $\rho_{1,0}=\rho_{0} Y_{1,0}, \rho_{2,0}=0$, and $\rho_{3,0}=\rho_{0}\left(1-Y_{1,0}\right)$.

\section{Discretization}

The model of the preceding sections must be solved numerically. Since the solutions will be used for calibration and sensitivity analysis, it is desirable to have a robust yet fast solver. To this end, the spatial temperature gradients are approximated as 2 nd order central differences with the temperature dependent thermal conductivities computed at cell boundaries. It was found that explicit Euler time advancement was stable using a reasonable time step typically on the order of $0.1 \mathrm{~s}$. In fact, the resultant code takes approximately half of a second of CPU time for a typical scenario as compared to around one hour for the implicit heat and mass transfer code of Kukuck. 
A common problem in the time integration of kinetic equations is the potential of predicting negative component densities. To prevent this numerical artifact, the reaction rate is set equal to the minimum of the physical rate and the rate that would bring the component density of the reactant species to zero over the current timestep. For example, the discretized equation for the bulk density of the dihydrate in computational cell $i$ is

$\rho_{1, i}^{(n+1)}=\rho_{1, i}^{(n)}-r_{1} \Delta t$

where

$r_{1}=\min \left\{A_{1} \rho_{1, i}^{(n)} \exp \left(-E_{1} / R T_{i}^{(n)}\right), \rho_{1, i}^{(n)} / \Delta t\right\}$

A similar relation was used for the rate of the second reaction.

\section{RESULTS}

In this section, the heat transfer model presented in the previous section is analyzed in terms of its consistency with experimental furnace test data from Takeda and Mehaffey [8]. The simulations assume nominal material properties taken from [5] as summarized in the second column of Table 2. The thermal conductivities, $k_{1}, k_{2}, k_{3}$, and $k_{4}$, correspond to values at temperatures of $0{ }^{\circ} \mathrm{C}, 175^{\circ} \mathrm{C}, 815{ }^{\circ} \mathrm{C}$, and $1200{ }^{\circ} \mathrm{C}$, respectively, where values at intermediate temperatures are determined by linear interpolation. The choice of these tabulation temperatures follows Kukuck [5], and they correspond to points at which the slope of the published thermal conductivity data for gypsum changes significantly. Kinetic parameters are calibrated to fit literature thermogravimetric analysis (TGA) data. Calibration of the heat transfer model parameters is performed by minimizing the sum of squared relative errors with respect to the experimental furnace test data. Finally, a local sensitivity analysis with respect to the calibrated model parameters is presented.

\section{Calibrating Reaction Kinetics from TGA}

Kinetic parameters $\left(A_{1}, E_{1}\right)$ and $\left(A_{2}, E_{2}\right)$ were calibrated using "Type X" gypsum board TGA data from Mehaffey et al. [3]. These data correspond to a heating rate of $20 \mathrm{~K} / \mathrm{min}$. The initial mass fraction of dihydrate was determined from the relative mass at $600 \mathrm{~K}$ to be $Y_{1,0}=0.846$. A fit was obtained by minimizing the sum of squared errors between the experimental data and the solution of the zero-dimensional mass conservation equations (Eqs. 4-6). The minimization was performed using the Nelder-Mead simplex algorithm [9].

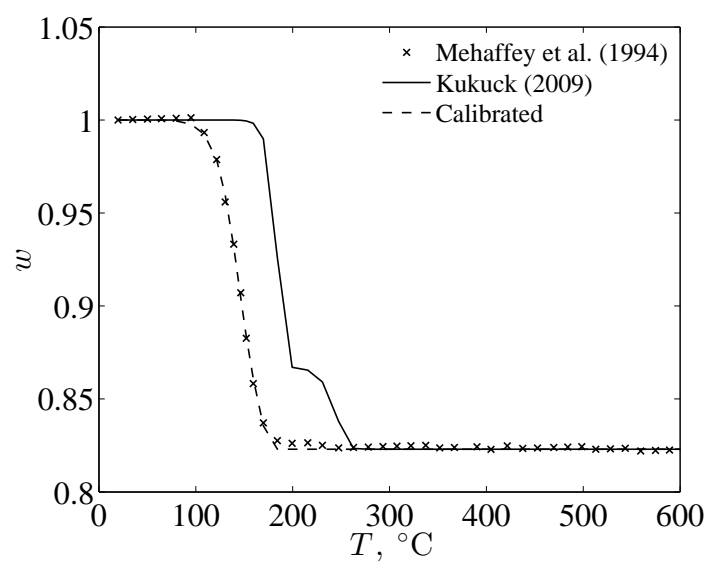

Fig. 4. Experimental and model TGA curves for "Type X”" gypsum board at a heating rate of $20 \mathrm{~K} / \mathrm{min}$.

A plot of the experimental TGA data along with model predictions using kinetic parameters from Kukuck [5] and those found by the calibration are shown in Fig. 4. The data is normalized by the initial mass, $m_{0}$, as $w(T) \equiv m(T) / m_{0}$. The literature and calibrated kinetic parameters are listed in Table 1 . Not only do the 
Table 1. Calibrated kinetic parameters.

\begin{tabular}{|l|c|c|}
\hline Parameter & Kukuck (2009) [5] & Calibrated \\
\hline \hline$A_{1}, 1 / \mathrm{s}$ & $3.56 \times 10^{30}$ & $5.56 \times 10^{12}$ \\
\hline$A_{2}, 1 / \mathrm{s}$ & $1.93 \times 10^{21}$ & $1.01 \times 10^{21}$ \\
\hline$E_{1}, \mathrm{~kJ} / \mathrm{mol}$ & 264 & 100 \\
\hline$E_{2}, \mathrm{~kJ} / \mathrm{mol}$ & 209 & 174 \\
\hline
\end{tabular}

calibrated parameters provide a better fit of the TGA data, but they also seem more reasonable. The preexponential of reaction 1 given by Kukuck is extremely large. Also, the calibrated activation energies have the expected ordering - the lower temperature reaction has the lower activation energy. For these reasons, the kinetic parameters calibrated to the data of Mehaffey et al. [3] will be used as nominal values in the remainder of this paper.

It should be noted that the TGA data of Mehaffey et al. indicate two other mass loss reactions not shown in Fig. 4. These reactions occur at temperatures of $650{ }^{\circ} \mathrm{C}$ and $1000{ }^{\circ} \mathrm{C}$. It is uncertain what these reactions are, but Ghazi Wakili et al. (2007) [10] suggest that an observed mass loss peak at $800{ }^{\circ} \mathrm{C}$ is attributable to calcium carbonate decomposition. The model used in this paper does not account for these reactions because the associated mass losses are relatively small (on the order of 3-4\% of total mass), and the heats of reaction are uncertain. However, the existence of these reactions should be noted as a possible source of model error.

In the next section, the calibrated kinetic parameters will be used as a starting point for exploring the ability of the heat transfer model to predict furnace test data.

\section{Model Parameter Calibration}

The plausibility of the heat transfer model was investigated by comparing simulation results to experimental furnace test data. Comparisons may be quantified by the sum of squared relative errors defined as

$e_{2}(\mathbf{y}) \equiv \frac{1}{N} \sum_{i=1}^{N}\left[\left(\frac{\hat{T}_{1 b}\left(t_{i} \mid \mathbf{y}\right)-T_{1 b, i}}{T_{1 b, i}}\right)^{2}+\left(\frac{\hat{T}_{2 b}\left(t_{i} \mid \mathbf{y}\right)-T_{2 b, i}}{T_{2 b, i}}\right)^{2}\right]$

where $\mathbf{y}$ is the vector of variable model parameters, $N$ is the number of times at which temperature comparisons are made, $\hat{T}\left(t_{i} \mid \mathbf{y}\right)$ is the model predicted temperature at time $t_{i}$ with model parameters equal to $\mathbf{y},\left(t_{i}, T_{i}\right)$ is the experimental data set, and the subscripts " $1 b$ " and " $2 b$ " denote temperatures at the backs of the first and second boards, respectively. Quantification of the model error helps to determine model plausibility in at least two respects. If $\mathbf{y}$ and $\left(t_{i}, T_{i}\right)$ are known with a high degree of certainty, then the model may be rejected if $e_{2}(\mathbf{y})$ is large-determinination of what constitutes a large error depends upon the required accuracy. For the case of the gypsum wallboard partition model of the previous section, this question can be asked by assuming that the nominal values listed in the second column of Table 2 are accurate. If $e_{2}\left(\mathbf{y}_{\text {nominal }}\right)$ is small, and the assumption of accurate nominal parameters is correct, this is strong evidence of a valid model.

In practice, there is some experimental uncertainty in the data, $\left(t_{i}, T_{i}\right)$, and typically a great deal of uncertainty associated with $\mathbf{y}$. Therefore, a second and less stringent test of model plausibility is whether the model error can be sufficiently small for at least one reasonable value of $\mathbf{y}$. This question may be answered by performing error minimization, or model calibration, over some reasonable space of $\mathbf{y}$. The error minimization problem is to find the set of model parameters, $\mathbf{y}^{*}$, that result in the minimum error, $e_{2}^{*}$. Formally stated, the calibration process will be to solve the optimization problem

$e_{2}^{*} \equiv e_{2}\left(\mathbf{y}^{*}\right)=\min _{\mathbf{y} \in \Omega} e_{2}(\mathbf{y})$

where $\Omega$ is the space of feasible model parameters and will vary depending upon the uncertainty in $\mathbf{y}$.

Solution of the calibration problem for several parameter spaces was performed using the open-source nonlinear optimization library NLopt [14]. Gradient-based methods were not considered for several reasons: the gradients of the objective function, $e_{2}$, are unknown; it is computationally expensive to compute finitedifferences; and it is likely that the objective function is not smooth. Two gradient-free algorithms were tried. FIRE SAFETY SCIENCE-PROCEEDINGS OF THE ELEVENTH INTERNATIONAL SYMPOSIUM pp. 598-611 
The Nelder-Mead Simplex method [9], is a robust algorithm for finding local minima, but the solution is strongly dependent on the initial guess for $\mathbf{y}$. Although the objective function, $e_{2}$, likely has many local minima, this algorithm was used as a first attempt since it is substantially faster than most global optimization algorithms. The second algorithm considered was controlled random search (CRS) with local mutation [15]. Because of its randomness, the CRS method can explore several local minima thereby increasing the probability of finding the true global minimum. For all cases, convergence was specified by relative tolerances in both $e_{2}$ and $\mathbf{y}$ of $1 \times 10^{-6}$, and the initial guess was taken to be the nominal property values listed in Table 2 . The best solutions, presented in Table 2, were obtained using the Nelder-Mead algorithm.

In addition to algorithm selection, appropriate bounds for the model parameters must be specified (the choice of $\Omega$ in Eq. 18). Three optimization cases were considered. The first problem was specified by fixing the parameters associated with mass loss $\left(\rho_{0}, Y_{1,0}, A_{1}, A_{2}, E_{1}\right.$, and $\left.E_{2}\right)$ and placing no constraints (other than physical constraints such as positive values for $k_{1}$, etc.) on the remaining 10 parameters. The justification for this is that the mass loss parameters are either known with a high degree of certainty or were obtained from experimental data. The decision to leave the remaining parameters unconstrained in this case was motivated by a lack of information and a desire to find the best possible solutions consistent with the model.

The second optimization problem formulation was similar to the first except that the number of unconstrained parameters was extended to 14 . In this case, only the initial density and mass fraction of dihydrate calcium sulfate were fixed. The kinetic parameters were relaxed because there is some uncertainty associated with the TGA calibration process described in the previous section. On the other hand, density and initial dihydrate mass fraction were kept fixed because there is little uncertainty associated with these measurements.

A third and final parameter space was used to investigate model plausibility. In this case, all 16 material properties were constrained to be within $\pm 10 \%$ of their nominal values. The choice of $10 \%$ was chosen because it represents a reasonable expectation of uncertainty in typical thermophysical property. Because this optimization problem is constrained, it is more likely to find solutions corresponding to physically realistic material property values.

Table 2. Heat transfer model calibration results.

\begin{tabular}{|l|c|c|c|c|}
\hline Property & Nominal & 10 Parameter & 14 Parameter & Constrained \\
\hline \hline$k_{1}, \mathrm{~W} / \mathrm{m} \cdot \mathrm{K}$ & 0.26 & 1.0 & 0.62 & $0.29(\mathrm{ub})$ \\
\hline$k_{2}, \mathrm{~W} / \mathrm{m} \cdot \mathrm{K}$ & 0.11 & 0.18 & 0.21 & $0.10(\mathrm{lb})$ \\
\hline$k_{3}, \mathrm{~W} / \mathrm{m} \cdot \mathrm{K}$ & 0.16 & 0.40 & 0.35 & $0.18(\mathrm{ub})$ \\
\hline$k_{4}, \mathrm{~W} / \mathrm{m} \cdot \mathrm{K}$ & 0.52 & 6.8 & 7.3 & $0.57(\mathrm{ub})$ \\
\hline$\rho_{0}, \mathrm{~kg} / \mathrm{m}^{3}$ & 648 & $\mathbf{6 4 8}$ & $\mathbf{6 4 8}$ & 631 \\
\hline$c_{p, 1}, \mathrm{~J} / \mathrm{kg} \cdot \mathrm{K}$ & 1080 & 55.4 & 54.0 & $972(\mathrm{lb})$ \\
\hline$c_{p, 2}, \mathrm{~J} / \mathrm{kg} \cdot \mathrm{K}$ & 803 & 4580 & 41.6 & 882 \\
\hline$c_{p, 3}, \mathrm{~J} / \mathrm{kg} \cdot \mathrm{K}$ & 703 & 5160 & 4640 & $773(\mathrm{ub})$ \\
\hline$\varepsilon$ & 0.9 & 1.0 & 1.0 & $0.99(\mathrm{ub})$ \\
\hline$Y_{1,0}$ & 0.846 & $\mathbf{0 . 8 4 6}$ & $\mathbf{0 . 8 4 6}$ & $0.93(\mathrm{ub})$ \\
\hline$A_{1}, 1 / \mathrm{s}$ & $5.56 \times 10^{12}$ & $\mathbf{5 . 5 6} \times \mathbf{1 0}^{12}$ & $2.78 \times 10^{11}$ & $5.00 \times 10^{12}(\mathrm{lb})$ \\
\hline$A_{2}, 1 / \mathrm{s}$ & $1.01 \times 10^{21}$ & $\mathbf{1 . 0 1} \times \mathbf{1 0}^{21}$ & $2.00 \times 10^{22}$ & $9.10 \times 10^{20}$ \\
\hline$E_{1}, \mathrm{~kJ} / \mathrm{mol}$ & 100 & $\mathbf{1 0 0}$ & 99 & $110(\mathrm{ub})$ \\
\hline$E_{2}, \mathrm{~kJ} / \mathrm{mol}$ & 174 & $\mathbf{1 7 4}$ & 188 & $191(\mathrm{ub})$ \\
\hline$\Delta h_{1}, \mathrm{~kJ} / \mathrm{kg}$ & 490 & 26.2 & 193 & $539(\mathrm{ub})$ \\
\hline$\Delta h_{2}, \mathrm{~kJ} / \mathrm{kg}$ & 209 & 1180 & 1240 & $230(\mathrm{ub})$ \\
\hline \hline$e_{2}^{*}$ & $3.47 \times 10^{-2}$ & $7.70 \times 10^{-4}$ & $6.21 \times 10^{-4}$ & $6.11 \times 10^{-3}$ \\
\hline$T_{2 b, 60}^{*},{ }^{\circ} \mathrm{C}$ & 193 & 115 & 117 & 129 \\
\hline
\end{tabular}

The results for the three calibration problem formulations are summarized in Table 2. The column headings "10 Parameter" and "14 Parameter" refer to the unconstrained minimizations, and "Constrained" refers to the parameter space with $\pm 10 \%$ bounds on all 16 parameters. For the unconstrained problems, the numbers in bold type correspond to fixed parameters. Active constraints in the constrained problem are specified by 
“(lb)" and "(ub)" for properties at their lower and upper bounds, respectively. In addition to the calibrated parameters, Table 2 also contains the sum of squared error of the solution, $e_{2}^{*}$, and the back wall temperature at $60 \mathrm{~min}, T_{2 b, 60}$. The experimental value of $T_{2 b, 60}$ from [8] is $111^{\circ} \mathrm{C}$.

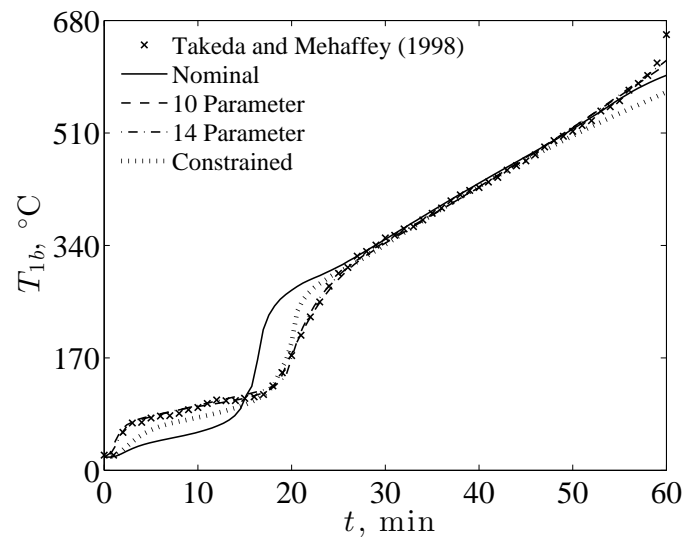

(a)

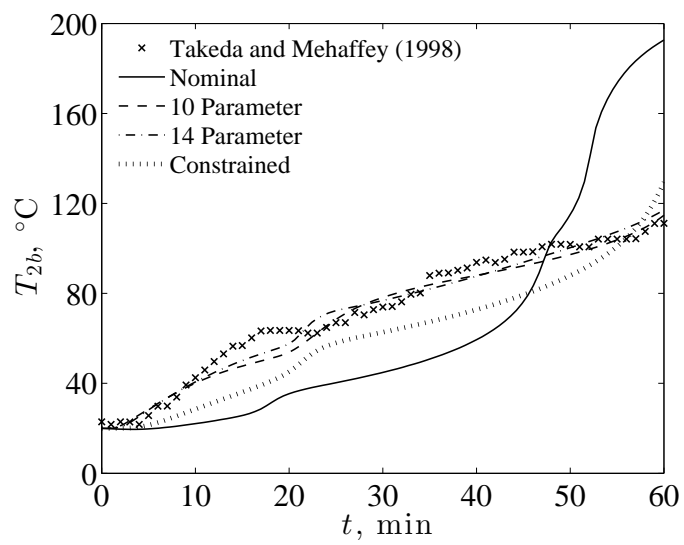

(b)

Fig. 5. Temperatures at the back of the exposed (a) and unexposed (b) gypsum boards using the heat transfer model with calibrated properties.

Plots of predicted back wall temperatures for the nominal and calibrated material properties are shown in Fig. 5. The predicted temperatures from the heat transfer model are plotted with the experimental data of Takeda and Mehaffey [8] that was used for calibration.

In discussing model plausibility, it was claimed that if the model predicts experimental data using the best estimates of the material properties (i.e., the nominal parameters) then the model is reasonable. Examination of Fig. 5 reveals that the model does not make an accurate prediction of the experimental data when it is parameterized with the nominal property values. In fact, the prediction of $T_{2 b, 60}$ using nominal properties is around $70{ }^{\circ} \mathrm{C}$ higher than the experimental data. This observation is not enough to invalidate the model since it is possible that nominal values are incorrect.

Inspection of Fig. 5 reveals that both the 10 and 14 unconstrained parameter optimizations result in nearly perfect predictions of $T_{1 b}$ and very good predictions of $T_{2 b}$ over most of the experimental times. As expected, the 14 parameter solution produces a slightly smaller value of $e_{2}$ since the kinetic parameters are allowed to vary. The dehydration activation energies in the 14 parameter case varied little from the nominal values found by calibration to TGA data. Note that in these unconstrained cases, several solution parameters assume unrealistic values. Therefore, it should not be presumed that the solutions (that is, the calibrated material properties) represent reasonable estimates of the actual material property values. However, the solutions might be useful in identifying specific weaknesses of the model. For example, the calibrated values of $k_{4}=k(1200$ ${ }^{\circ} \mathrm{C}$ ) in the unconstrained problems are up to 13 times larger than the nominal value. Although the maximum experimental temperature in the exposed board is $927{ }^{\circ} \mathrm{C}, k_{4}$ has a significant influence on the temperature predictions because the model uses linear interpolation to compute $k$ between $815^{\circ} \mathrm{C}$ and $1200{ }^{\circ} \mathrm{C}$ (the temperatures at which $k_{3}$ and $k_{4}$ are defined). An explanation for the calibration finding a large high temperature thermal conductivity might be attributable to porous media radiation in which $k \propto T^{3}$.

Another surprising calibration result for the 10 and 14 parameter unconstrained cases was that the heat capacities for the dihydrate calcium sulfate were estimated to be approximately 20 times smaller than the measured value. Since the measurement of heat capacity at room temperature is a relatively straightforward measurement, the predicted low values for $c_{p, 1}$ indicate a modeling error. The calibrated heat capacities of the anhydrous calcium sulfate, $c_{p, 3}$, were approximately 6.6 times larger than the nominal value. Since the anhydrous state only becomes dominant at higher temperatures, this result could be a consequence of the assumption of constant heat capacities. Practically speaking, future models should include temperature de- 
pendent heat capacities. Another possible explanation for the over-estimate of $c_{p, 3}$ is that the optimization is attempting to account for the heat of decomposition of calcium carbonate that was not included in the model.

The calibrated values of the heats of dehydration are also significantly different from the nominal values. From the TGA data in Fig. 4 it is seen that the two dehydration reactions are visually indistinguishable. Therefore, it is likely that the two reactions could be lumped together and produce similar results in the heat transfer model. If this is the case, then the more appropriate quantity for comparison of the calibrated and nominal heats of dehydration is the total heat of dehydration, $\Delta h_{1}+\Delta h_{2}$. The calibrated total heats of dehydration are about twice as large as the nominal. This observation is possibly a compensation for the low heat capacities calibrated for dihydrate $\mathrm{CaSO}_{4}$.

It was found that there were many local minima for the 14 parameter problem. The first several optimization attempts found minima with larger values of $e_{2}$ than were found in the 10 parameter problem. Therefore, it is probable that the results presented in Table 2 are not global minima. It is possible that better fits of the data exist with more reasonable values of the model parameters. Instead of spending an indefinite amount of time trying to find these solutions in the unconstrained space, a constrained optimization was attempted.

The fit of the constrained $\pm 10 \%$ solution is relatively good: the error is half of the nominal but about 10 times more than the unconstrained solutions. However, in terms of the principal quantity of interest for fire resistance problems, $T_{2 b, 60}$, the error is significant. The back wall temperature at $60 \mathrm{~min}$ is $18{ }^{\circ} \mathrm{C}$ larger than the experimental value. It is unlikely that this is within the experimental error.

The results of this section indicate several possible model improvements. It is apparent that the proposed heat transfer model can come close to predicting experimental furnace test data, but it is unclear whether accurate model predictions can be accomplished with reasonable material property values. Consequently, more information is needed on the various model parameters and their uncertainties. If the nominal material properties assumed in the preceding analysis turn out to be mostly correct, then the model improvements indicated by the calibration results will need to be implemented. To help identify which material properties are most influential to the model output, a sensitivity analysis is presented in the next section.

\section{Local Sensitivity Analysis}

An understanding of model sensitivity can help to guide future modeling and experimental work. The sensitivities of the back surface temperature at $60 \mathrm{~min}, T_{2 b, 60}$, with respect to all 16 model parameters were computed according to the first order finite-difference approximation of Eq. 3. The parameter perturbations were decreased until the finite-differences converged, and the results are summarized in Fig. 6 for the material properties corresponding to the four columns in Table 2. Multiple locations in the parameter space were studied in order to understand how the sensitivities vary over the parameter space. For example, if the nominal material properties are significantly incorrect, the sensitivities computed about the nominal material might be significantly incorrect. By considering multiple points in the parameter space, it is possible to observe some general trends in the local sensitivities.

For all four material property descriptions, density is one of the top two most influential parameters. In all cases these sensitivities are negative since the final back wall temperature decreases with increasing density. This is not surprising since the thermal inertia of the boards is directly proportional to the amount of mass present. Evidently, increasing density of gypsum wallboards is the most effective means to increasing fire resistance. Unfortunately, this runs counter to the design goal of decreasing the weight of the wallboards. The next most consistently influential parameter is the thermal conductivity at $815^{\circ} \mathrm{C}, k_{3}$. Recall that, because of the linear interpolation on thermal conductivity values, the value of $k_{3}$ affects heat conduction at all temperatures between $175^{\circ} \mathrm{C}$ and $1200{ }^{\circ} \mathrm{C}$. 


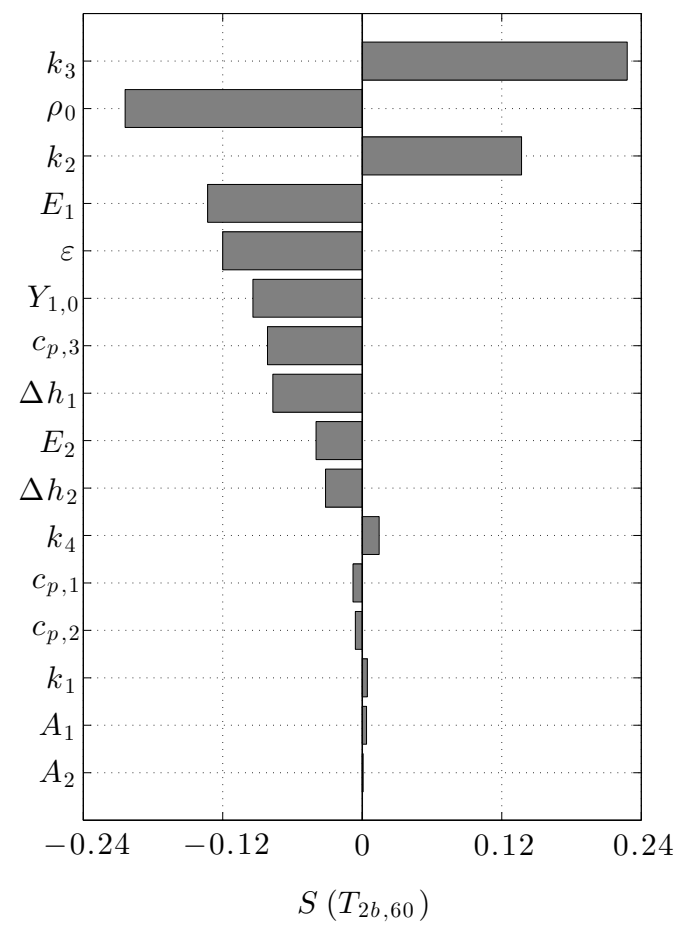

(a)

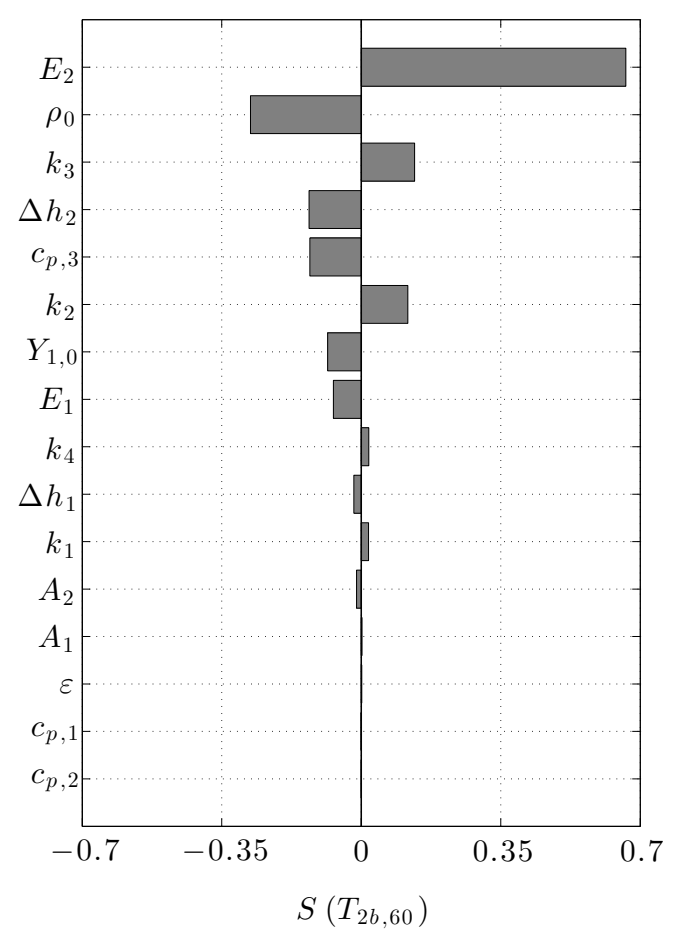

(c)

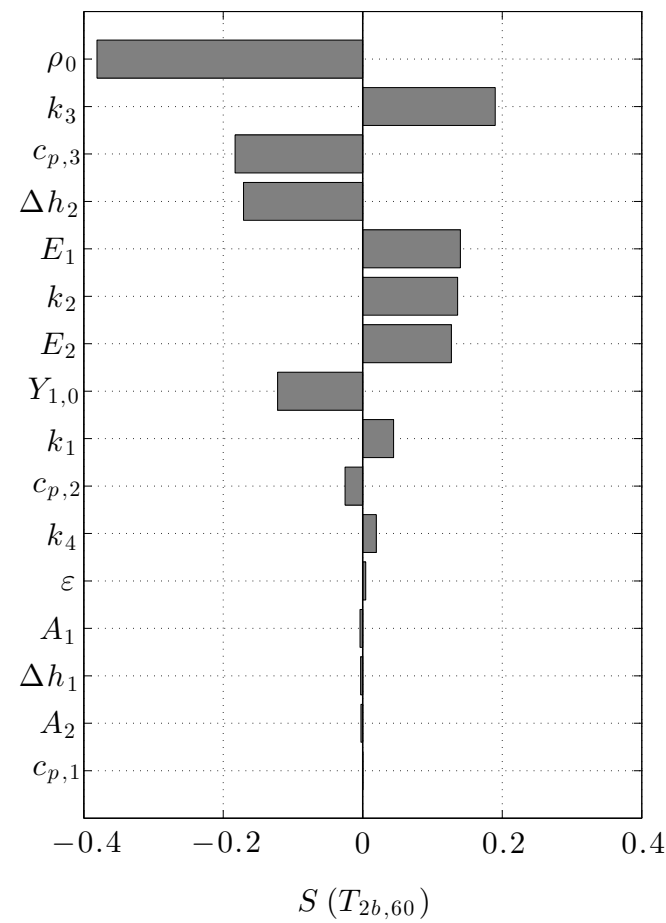

(b)

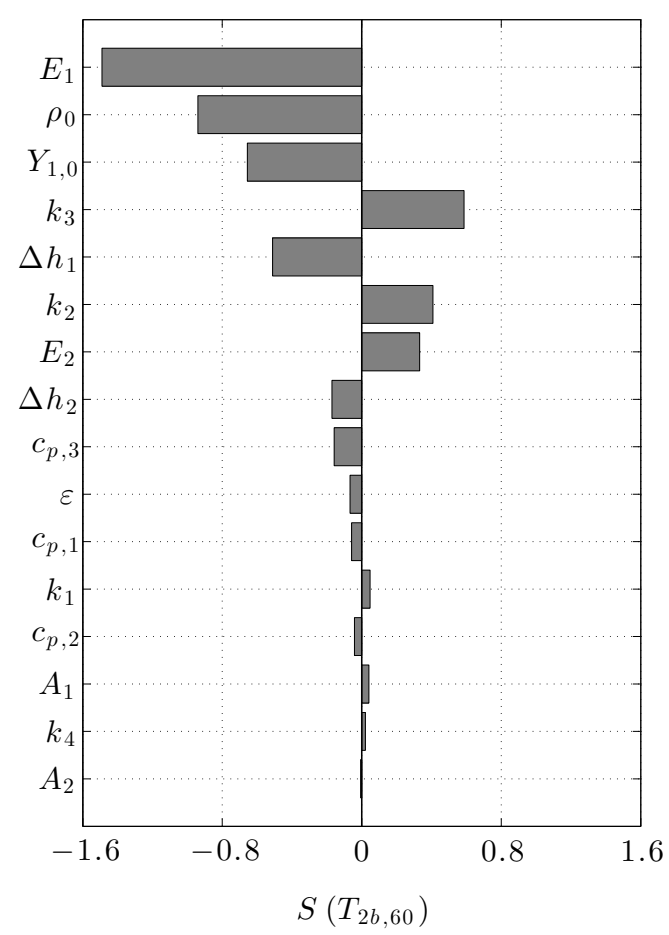

(d)

Fig. 6. Heat transfer model sensitivities at (a) nominal material properties, (b) 10 parameter, (c) 14 parameter, and (d) fully constrained calibration material properties. 
Another consistently influential parameter is the activation energy of the first dehydration reaction. Unlike the sensitivities with respect to density and thermal conductivity, the sensitivity with respect to $E_{1}$ changes sign throughout the parameter space. The backwall temperature seems to be less sensitive to the activation energy of the second dehydration reaction, but $E_{2}$ is moderately important in all four cases. The sensitivity of the model to the activation energies is probably a mathematical artifact in that each $E_{j}$ is inside an exponential function. This does not change the fact that the model results are very sensitive to the values of $E_{1}$ and $E_{2}$, but it does not necessary imply that the model is sensitive to the rates of reaction.

Among the least influential prameters are the dehydration reaction pre-exponentials, $A_{1}$ and $A_{2}$. Additionally, the sensitivities with respect to $c_{p, 1}$ and $c_{p, 2}$ are generally much smaller than the sensitivities to $c_{p, 3}$. This dominance is explained by the fact that the model assumes that the material is mostly composed of anhydrous calcium sulfate, and that at high temperatures the wallboard will be entirely anhydrous. The model sensitivities with respect to heat capacities are always negative since increasing heat capacity increases thermal inertia.

\section{CONCLUSIONS}

The effectiveness of a pure heat transfer model of a gypsum wallboard partition furnace test was investigated. Simulation and sensitivity analysis of an existing heat/mass transfer model indicates that mass transfer and condensation of water vapor are not important physical processes for predicting board temperatures in a typical ASTM E119 furnace test. This agrees with similar observations from the literature. A computationally efficient model of heat transfer in a gypsum partition furnace test was developed. Nominal parameters were taken from the literature or calibrated against literature TGA data. Additional model parameters were calibrated against literature furnace test data. The calibrations were performed over three separate feasible parameter spaces using two gradient-free optimization algorithms. The proposed model could not be validated or invalidated due to uncertainty in the model parameters, but the results of the calibrations indicated several areas for model improvement. To identify which parameters need to be better characterized, local sensitivity analyses were performed about the nominal and calibrated material property sets. Several properties were seen to be consistently influential, and several properties were seen to be consistently uninfluential. In summary, successful predictions of heat transfer in gypsum wallboard partitions will require improvements in modeling and material property measurements. Suggested model improvements are the addition of a calcium carbonate decomposition reaction, temperature dependent specific heat capacities, and a porous media radiation model for thermal conductivity. Suggested improvements in material property measurements include precise density measurements and thorough kinetic parameter calibrations against TGA data.

In addition to improving the model and better characterizing material properties, several other issues must be addressed before reliable performance-based design of gypsum wallboard partitions is possible. In particular, scenario parameter uncertainty must be investigated. The exposed surface boundary condition is not adequately characterized, and it is possible that there is significant error accompanying the assumption of an exposed surface temperature boundary condition. One approach to resolving this problem is to couple a CFD model of the furnace to the heat transfer model. Also, the model of the air gap is fairly simple, and it is important to investigate the effects of fluid flow and heat conduction through wall studs have on the back wall temperature. Another complicating factor is the existence of combustible paper on the surface of the gypsum boards. Because the paper is relatively thin this might be unimportant, but it is relatively easy to study within the context of a one-dimensionl heat transfer model. Finally, if the suggested model improvements of the preceding paragraph do not result in a well-validated model, it will be necessary to reinvestigate the importance of water vapor mass transfer and condensation.

\section{ACKNOWLEDGMENTS}

This research was performed while one of the authors (M. Bruns) held a National Institute of Standards and Technology (NIST) Research Postdoctoral Associateship Award at the Engineering Laboratory. Funding for the award was provided by NIST. 


\section{REFERENCES}

[1] American Society for Testing and Materials. Standard Test Methods for Fire Tests of Building Construction and Materials. ASTM E119-12a.

[2] Bukowski, R. W. Prediction of the Structural Fire Performance of Buildings. In Eighth Fire and Materials Conference, San Francisco, 2003.

[3] Mehaffey, J. R. and Cuerrier, P. and Carisse, G., (1994) A Model for Predicting Heat Transfer through Gypsum-Board/Wood-Stud Walls Exposed to Fire, Fire and Materials 18: 297-305, http: //dx.doi.org/10.1002/fam.810180505

[4] Sultan, M. A. (1996) A Model for Predicting Heat Transfer through Noninsulated Unloaded SteelStud Gypsum Board Wall Assemblies Exposed to Fire, Fire Technology 32: 239-259, http://dx.doi. org/10.1007/BF01040217

[5] Kukuck, S., "Heat and Mass Transfer Through Gypsum Partitions Subjected to Fire Exposures," National Institute of Standards and Technology Report NISTIR 7461, Gaithersburg, MD, 2009.

[6] Manzello, S. L. and Gann, R. G. and Kukuck, S. R. and Prasad, K. and Jones, W. W., (2007) Performance of a Non-Load-Bearing Steal Stud Gypsum Board Wall Assembly: Experiments and Modelling, Fire and Materials 31: 297-310, http://dx.doi.org/10.1002/fam.939

[7] Weber, B. (2012) Heat Transfer Mechanisms and Models for Gypsum Board Exposed to Fire, International Journal of Heat and Mass Transfer 55: 1661-1678, http://dx.doi.org/10.1016/j. ijheatmasstransfer.2011.11.022

[8] Takeda, H. and Mehaffey, J. R. (1998) WALL2D: a Model for Predicting Heat Transfer through Wood-Stud Walls Exposed to Fire, Fire and Materials 22: 133-140, http://dx.doi.org/10.1002/ (SICI)1099-1018(1998070)22:4〈133::AID-FAM642〉3.0.CO;2-L

[9] Nelder, J. A. and Mead, R., (1965) A Simplex Method for Function Minimization, The Computer Journal 7: 308-313, http://dx.doi.org/10.1093/comjn1/7.4.308

[10] Ghazi Wakili, K. and Hugi, E. and Wullschleger, L. and Frank, T., (2007) Gypsum Board in FireModeling and Experimental Validation, Journal of Fire Sciences 25: 267-282, http://dx.doi.org/10. $1177 / 0734904107072883$

[11] Sultan, M. A. and Benichou, N. and Min, B. Y. Heat Exposure in Fire Resistance Furnaces: Fullscale vs. Intermediate-Scale. In Eighth Fire and Materials Conference, San Francisco, 2003.

[12] ElSherbiny, S. M. and Raithby, G. D. and Hollands, K. G. T., (1982) Heat Transfer by Natural Convection Across Vertical and Inclined Air Layers, Journal of Heat Transfer 104: 96-102, http: //dx.doi.org/10.1115/1.3245075

[13] Churchill, S. M. and Chu, H. H. S., (1975) Correlating Equations for Laminar and Turbulent Free Convection from a Vertical Plate, International Journal of Heat and Mass Transfer 18: 1323-1329, http://dx.doi.org/10.1016/0017-9310(75)90243-4

[14] Johnson, S. G., (2013) The NLopt nonlinear-optimization package, http://ab-initio.mit.edu/nlopt

[15] Kaelo, P. and Ali, M. M., (2006) Some Variants of the Controlled Random Search Algorithm for Global Optimization, Journal of Optimization Theory and Applications 130: 253-265, http: //dx.doi.org/10.1007/s10957-006-9101-0 